\title{
Colonic duplication in adulthood presenting with diarrhea
}

In pediatric clinics, alimentary tract duplications are seen with an incidence of approximately 1 in 4500 [1]. Most cases are diagnosed within the first 5 years of life [2]. The condition is seen infrequently in adults. Patients usually present with constipation, diarrhea, abdominal pain, abdominal mass, and, sometimes, acute abdomen, volvulus, or perforation [3]. There are two types of colonic duplication: tubular and cystic. Diagnosis requires a careful history and detailed imaging with endoscopy, computed tomography, and in some cases barium meal study. Correct diagnosis is important not only because the symptoms decrease patients' quality of life, but also because with time patients are at increased risk of developing carcinoma [4]. The most common form of carcinoma associated with alimentary tract duplication is adenocarcinoma.

A 39-year-old man without any other history of chronic disease was referred to our clinic because of chronic diarrhea. He described the watery diarrhea as having been present for 6 months and being independent of eating. He had no fever, rectal bleeding, or tenesmus. He complained of having had irregular abdominal pain since childhood. Physical examination showed no significant findings. The work-up for ruling out infectious causes, including microscopic examination for protozoa and cultures of the feces, produced negative findings. Abdominal radiography was performed but showed nothing significant, and colonoscopy was done in order to rule out inflammatory bowel disease and celiac disease. At colonoscopy, thick, band-like mucosal lesions were seen dividing the lumen into three spaces, like the Mercedes logo, with wide exudative superficial ulcers and polypoid lesions on top of and around them ( $\bullet$ Fig.1). Biopsy results showed lowgrade dysplasia on the malformation site (৫ Fig.2), and the patient was referred for surgery. Low anterior resection was performed. The symptoms regressed after surgery and the follow-up colonoscopy after 6 months showed no significant findings.

Intestinal duplications are rare gastrointestinal abnormalities in adulthood. They are defined as mucosal structures with smooth muscle lining which form another lumen (tubular duplication) or cysts (cystic duplication). The cystic form is the most commonly found [5]. Colonic duplication is one of the rarest types of duplication of the alimentary tract [6]. Its pathogenesis is still unclear, but there are several theories relating it to malformation of the embryonic gut. Some authors suggest that environmental factors may also have a role.

Although the disease is usually rapidly diagnosed in infants, diagnosis can be challenging in adults. Manifestations usually include pain, obstructive symptoms, and sometimes mass lesions. With its broad range of symptoms, this abnormality can be difficult to differentiate from infections, inflammatory bowel disease, gastrointestinal bleeding, or obstruction. In our case the patient presented with chronic diarrhea. To our knowledge, this is the first reported case of colonic duplication presenting with diarrhea as the main symptom.

Another aspect that needs to be underlined about colonic duplication is that it can lead to carcinoma, most importantly adenocarcinoma. In a series of seven cases of colorectal duplication, one patient had high-grade dysplasia and one had low-

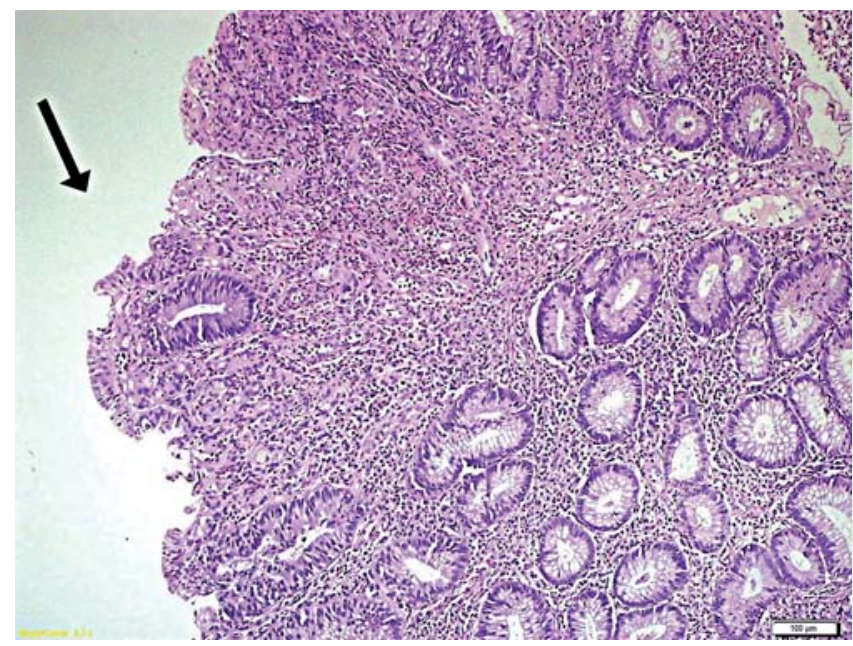

Fig. 2 Photomicrograph showing dysplasia on the site of the colonic malformation (arrow).
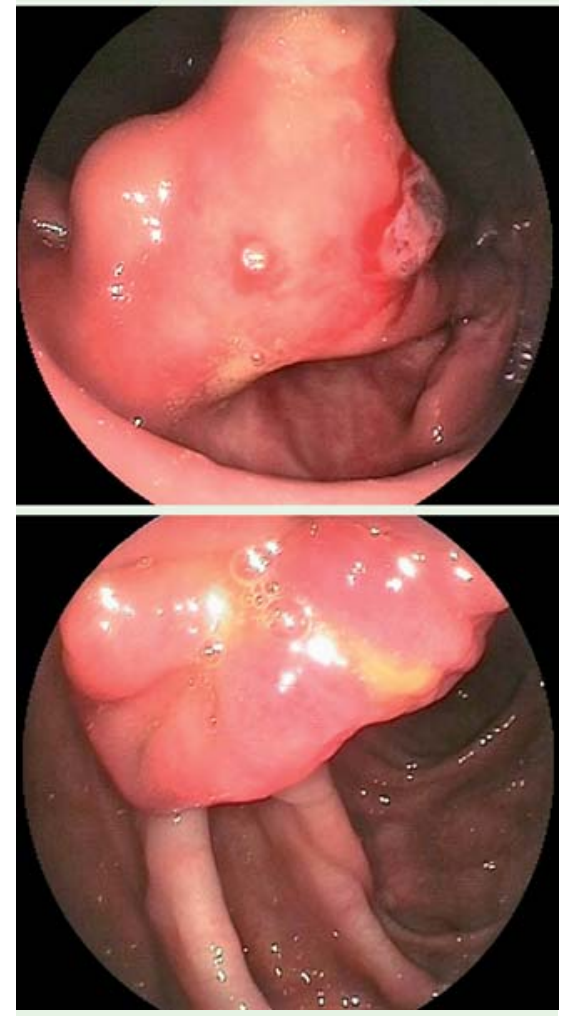

Fig. 1 Endoscopic images showing colonic duplication and ulcers in a 37-year-old man. 
grade dysplasia, as did our patient [7]. In one published case, the patient presented with metastasis to the omentum [8]. In order to prevent progression to adenocarcinoma, en bloc resection of the colon is suggested as soon as possible once the diagnosis of colonic duplication is made. Colonic duplication in adulthood is very rare, but it should be kept in mind in the differential diagnosis of chronic diarrhea and abdominal pain. If overlooked, it may result in malignancy.

Endoscopy_UCTN_Code_CCL_1AD_2AJ

\section{Competing interests: None}

\section{Ozge Telci Caklili'1,2, ilyas Tuncer², Yasar Colak ${ }^{2}$, Duygu Kosemetin ${ }^{3}$, Ayşe Bahar Ceyran ${ }^{3}$}

${ }^{1}$ Department of Internal Medicine, Istanbul Medeniyet University Goztepe Training and Research Hospital, Istanbul, Turkey
2 Department of Gastroenterology, Istanbul Medeniyet University Goztepe Training and Research Hospital, Istanbul, Turkey

${ }^{3}$ Department of Pathology, Istanbul Medeniyet University Goztepe Training and Research Hospital, Istanbul, Turkey

\section{References}

1 Puligandla PS, Nguyen L, St-Vil D et al. Gastrointestinal duplications. J Pediatr Surg 2003; 38: 740-744

2 Correia-Pinto J, Romero R, Carvalho JL et al. Neonatal perforation of a Y-shaped sigmoid duplication. J Pediatr Surg 2001; 36: 1422 1424

3 Ho YC. Total colorectal and terminal ileal duplication presenting as intussusception and intestinal obstruction. World J Gastroenterol 2012; 18: 6338-6340

4 Kekez T, Augustin G, Hrstic I et al. Colonic duplication in an adult who presented with chronic constipation attributed to hypothyroidism. World J Gastroenterol 2008; 14: 644-646

5 Soares-Oliveira M, Carvalho JL, Campos M et al. Intestinal duplication. A diagnostic and therapeutic challenge. Acta Med Port 2002; 15: $365-368$
6 Lund DP. Alimentary tract duplications. In: Grosfeld JL, O'Neill JA, Fonkalsrud EW, Coran AG, eds. Paediatric Surgery. 6th ed. Philadelphia: Mosby Elsevier; 2006: 1389-1399

7 Mourra N, Chafai N, Bessoud B et al. Colorectal duplication in adults: report of seven cases and review of the literature. J Clin Pathol 2010; 63: 1080-1083

$8 \mathrm{Hsu} H$, Gueng $M K$, Tseng $Y H$ et al. Adenocarcinoma arising from colonic duplication cyst with metastasis to omentum: a case report. J Clin Ultrasound 2011; 39: 41-43

\section{Bibliography}

Dol http://dx.doi.org/

10.1055/s-0033-1358805

Endoscopy 2013; 45: E430-E431

(C) Georg Thieme Verlag KG

Stuttgart · New York

ISSN 0013-726X

\section{Corresponding author}

\section{Ozge Telci Caklili, MD}

Gastroenterology, Department of Internal Medicine

Istanbul Medeniyet University Goztepe Research and Training Hospital

Fahrettin Kerim Gokay Caddesi

Kadıkoy Istanbul 34730

Turkey

Fax: +90-216-5664023

wattersonx@gmail.com 